\title{
Educação ambiental na construção de políticas para a gestão dos resíduos em uma instituição de ensino superior ${ }^{1}$
}

\author{
Luciara Bilhalva Corrêa² \\ Valéria Lerch Lunardi ${ }^{3}$ \\ Pedro Roberto Jacobi ${ }^{4}$
}

\begin{abstract}
RESUMO: Com o objetivo de construir políticas de gestão dos resíduos numa universidade federal, na dimensão da educação ambiental, realizou-se estudo qualitativo, na modalidade pesquisa-ação. Mediante análise de conteúdo dos dados, emergiu a categoria, "A aparente invisibilidade do processo de construção de políticas para a gestão dos resíduos na IES", enfocando a necessidade de conhecer a estrutura e a realidade institucional, a geração e condições de manejo de resíduos nas suas diferentes unidades. A educação ambiental potencializou a comunidade universitária a pensar a realidade institucional como um sistema complexo integrado, contribuindo para um agir ético e comprometido com a sustentabilidade.
\end{abstract}

Palavras-chave: universidades; gestão de resíduos; sustentabilidade.

\section{INTRODUÇÃO}

As atividades desenvolvidas nas Instituições de Ensino Superior - IES produzem diferentes tipos de resíduos, caracterizando-se como uma matriz complexa e heterogênea com impactos negativos no ambiente quando gerenciados de forma inadequada.

Apesar de as IES serem centros promotores de discussão ambiental, na maioria das vezes, sua comunidade não atenta de forma responsável para a poluição gerada em seus âmbitos. Em muitas situações, exibem práticas contraditórias aos seus princípios, ou seja, ainda revelam uma visão autocentrada, fragmentada e desconectada dos reais desafios socioambientais (DIAS, 2006).

A gestão dos resíduos não está atrelada somente a aspectos legais e tecnológicos, seu maior desafio implica a dimensão ética, de responsabilidade da sociedade, vinculado a um processo educativo permanente.

A adoção de políticas para a gestão dos resíduos nas IES pode ser observada em vários estudos, especialmente nos de De Conto et al. (2004). Entretanto, são escassos aqueles que privilegiem uma proposta educativa para potencializar os sujeitos que compõem estes ambientes, na construção conjunta de políticas ambientais para a gestão dos resíduos; quando contemplam ações educativas, suas propostas, de modo predominante, são tradicionais, verticalizadas e pontuais, baseadas num modelo rígido a partir de disponibilização de informações sobre técnicas de gerenciamento, priorizando condutas para atender as etapas do manejo de resíduos.

\footnotetext{
1 Artigo elaborado a partir da Tese de doutorado apresentada ao Programa de Pós-Graduação em Educação Ambiental da Universidade Federal do Rio Grande - FURG.

2 Doutora em Educação Ambiental. Professora Adjunta da Universidade Federal de Pelotas - UFPEL. E-mail: <luciarabc@gmail.com.br>.

3 Doutora em Enfermagem. Professora do Programa de Pós-Graduação em Educação Ambiental e do Departamento de Enfermagem da Universidade Federal do Rio Grande - FURG. E-mail: <vlunardi@terra.com.br>.

4 Professor Titular da Faculdade de Educação e do Programa de Pós Graduação em Ciência Ambiental da Universidade de São Paulo - USP. E-mail: <prjacobi@usp.br>.
} 
Nessa perspectiva de aparente exclusão, "são observadas múltiplas formas de resistência da comunidade universitária em participar nos programas de gestão dos resíduos na instituição, demonstrando um descompromisso dos sujeitos em relação às ações propostas, justamente por não terem se envolvido com o processo" (DIAS, 2001, p. 37).

Daí a importância da construção de políticas para a gestão dos resíduos em IES, a partir de um enfoque da educação ambiental, numa perspectiva sistêmica, para um pensar complexo. Considera-se inicialmente, o envolvimento articulado dos diferentes indivíduos que compõem o ambiente universitário, na busca de uma nova relação para as questões dos resíduos em seu contexto. A política estabelece objetivos ambientais estratégicos na organização, a partir de discussão interna da qual participam seus dirigentes, funcionários, colaboradores, a comunidade acadêmica como um todo (CORRÊA, 2009).

Assim, este artigo tem como objetivo apresentar o processo educativo na dimensão da educação ambiental na construção de políticas integradas para a gestão dos resíduos em uma IES.

\section{METODOLOGIA}

Trata-se de pesquisa qualitativa, na modalidade da pesquisa-ação (THIOLLENT, 2008), realizada na Universidade Federal de Pelotas - UFPEL, localizada no sul do Brasil. Participaram 28 sujeitos entre os quais gestores, funcionários, professores e alunos. Foram realizados grupos focais; estudos documentais e observação do ambiente institucional. A partir da análise de conteúdo, proposto por Moraes (2003), emergiram duas categorias na pesquisa. A primeira, apresentada a seguir, considerada relevante, segundo Morin (2003), por potencializar gradativamente transformações no pensamento dos sujeitos, ainda que a evolução do processo proposto tenha predominantemente se mantido numa aparente invisibilidade.

\section{AAPARENTE INVISIBILIDADE DO PROCESSO DE CONSTRUÇÃO DE POLÍTICAS PARA A GESTÃO DOS RESÍDUOS NA INSTITUIÇÃO DE ENSINO SUPERIOR}

Tratou-se de primeira tentativa da administração superior da IES de possibilitar um espaço de diálogo coletivo para a discussão da gestão de resíduos no contexto institucional. Foi constituído o Grupo Desencadeador (GD) com diferentes sujeitos da comunidade com o propósito de iniciar propriamente o processo de construção de políticas para a gestão dos resíduos, mediante a explicitação, nesse coletivo, do conhecimento das suas intenções, percepções, vivências, desejos e perspectivas, contribuindo para o avanço e emergência de aspectos importantes nessa caminhada.

Foi fundamental um processo pedagógico que potencializasse a comunidade universitária participante a conhecer e pensar sua realidade como um sistema organizado, e não isolado e ainda atrelado a uma visão de mundo da ciência clássica: nesta visão o objeto existe de maneira positivista, sem que o sujeito participe da sua construção, através das estruturas de seu entendimento (MORIN, 2005a). Deste modo, o processo foi permeado de indagações: Como é sua realidade quanto aos resíduos e seu processo de manejo? No ambiente institucional, como vem sendo tratada essa questão? Qual nosso papel frente essa situação?...( $\left(\mathrm{GD}_{22}\right)$.

Este caminho percorrido pelo GD, aparentemente invisível, foi considerado pelo próprio grupo como necessário e importante, como processo de aprendizagem. Os sujeitos participantes manifestavam desejo de conhecer os objetivos da proposta pedagógica na perspectiva da educação ambiental, os motivos do apoio da administração superior na constituição desse grupo, os sujeitos que o compunham, suas vivências, fragilidades,

Revbea, Rio Grande, 7: 9-15, 2012. 
dificuldades, possibilidades e potencialidades para um processo de construção de políticas para a gestão dos resíduos na Instituição.

A intenção do processo educativo foi incentivar a inclusão e valorização dos sujeitos envolvidos, em que todos pudessem ser educadores, mediante trocas de experiências e saberes, descobrindo como construir políticas para a gestão dos resíduos na instituição, reintroduzindo o papel do sujeito observador, conceituador, estrategista em todo o conhecimento: uma construção que é sempre incerta, porque o sujeito se encontra inserido na realidade que pretende conhecer (MORIN, CIURANA, MOTA, 2003; QUINTAS, GOMES, UEMA, 2005).

O processo foi permeado de desafios, pela necessidade de aprendizagem da construção e interação junto ao coletivo, em uma estrutura institucional que culturalmente dialoga pouco, por constituir-se fragmentada e departamentalizada. Limites e dificuldades foram identificados, especialmente pela visão de mundo dos sujeitos envolvidos, que pareciam esperar, passivamente, a solução imediata e o estabelecimento de ordem à aparente desordem provocada pela emersão do problema dos resíduos no contexto institucional: ...Agora vai ter alguém para resolver nosso problema dos resíduos; é tudo contigo; chegaste na hora certa; porque a situação está problemática, e não sabemos o que fazer, nem temos gente que cuide disso aqui... $\left(G_{1}\right)$.

Essa visão, entretanto, não representava problemas nem limites para a continuidade do processo educativo, visto que esse movimento de desconstrução, reconstrução, desafiava-nos, pois uma reforma no pensamento ocorre na ruptura e na transformação dos sujeitos, em aprendizagem permanente.

Houve no grupo uma constante problematização da situação dos resíduos no ambiente da Instituição, como seu manejo inadequado; a falta de um programa de gestão que incluísse suas diferentes etapas, o descomprometimento, a falta de percepção e de valor em relação ao ambiente e aos resíduos, a necessidade da gestão dos resíduos nas diferentes atividades da instituição, dentre outros.

Como os sujeitos focavam suas preocupações no âmbito da sua unidade de atuação, sua fala demonstrava limites em colaborar com o processo de pensar estratégias para a construção de políticas para a gestão dos resíduos ao todo institucional: ...Eu não tenho formação na área de meio ambiente, então, não tenho como colaborar com o grupo na questão dos resíduos aqui da Instituição. O que posso dizer é que faltam lixeiras aqui, disponibilizar isso resolve a maioria dos problemas em relação ao lixo; sinceramente não vejo no que posso contribuir a mais nessa questão... $\left(\mathrm{GD}_{24}\right)$.

Esse depoimento mostra a necessidade e o desafio de conduzir um processo educativo na perspectiva da complexidade, por demonstrar limites, insuficiências e carências de um pensamento simplificador. O desafio da complexidade é sensibilizar para as carências do nosso pensamento, e compreender que um pensamento mutilador conduz necessariamente a ações mutilantes. Portanto, as práticas pedagógicas precisam ser um exercício para que o pensamento seja capaz de lidar com o real, de com ele dialogar e negociar.

O movimento de interação mobilizou o grupo para o desencadeamento da estratégia de conhecer a estrutura institucional, relacionando o todo com a parte onde atuavam, potencializando o entendimento de que, no ambiente institucional como um sistema organizado, tudo está ligado e em relação (MORIN, 2006).

Nessa perspectiva, a proposta foi desafiar o Coordenador do GD a apresentar o contexto Institucional, pois os participantes manifestavam desconhecimento do todo da instituição, consequentemente, com maior possibilidade do exercício de ações irresponsáveis com o ambiente, seja quanto à problemática dos resíduos como do contexto onde essa problemática se inseria: ...Eu nem sabia para onde vai nosso lixo... Capaz que ele é jogado aqui dentro da Instituição, nossa... geramos lixo todos os dias, vem a moça da limpeza junta o saco e leva... Não imaginava que era assim... (GD $\left.{ }_{9}\right)$.

Revbea, Rio Grande, 7: 9-15, 2012. 
A caminhada no processo de construção intensificava a identidade crescente do grupo com a educação ambiental, mobilizando espaços em que a participação fosse assumida e valorizada com a discussão e reflexão sobre seu ambiente, potencializando a transformação individual e coletiva, baseada em valores éticos e comprometidos com o ambiente (JACOBI, 2005).

O movimento promovido no grupo a partir da apresentação da estrutura institucional, também encaminhou a uma necessidade ética de diálogo com sujeitos de outros setores e unidades, da sua presença e participação no processo de aprendizagem para a construção de políticas para a gestão dos resíduos, enriquecendo discussões e decisões, visando uma gestão sustentável dos resíduos na perspectiva sistêmica: ...o prefeito da Instituição precisa estar aqui para junto conosco pensar em possibilidades de ação. Ele é parte fundamental nesse processo, porque ele coordena setor de limpeza, manutenção, obras; como planejar sem ele?......Assim como, a necessidade de outros profissionais, como o de segurança do trabalho, porque ele conhece e sabe avaliar riscos; o do setor de comunicação, porque ele é fundamental na divulgação dessas políticas... $\left(\mathrm{GD}_{25}\right)$.

Morin (2005b, p. 64) considera que o "pensamento complexo alimenta a ética. Ao religar os conhecimentos, orienta para a religação entre seres humanos com o contexto planetário", uma vez que revela e incentiva o aparecimento de uma nova consciência coletiva capaz de evitar a destruição do planeta. Este pensamento também conduz para uma ética da responsabilidade, da solidariedade, do exercício da cidadania e da sustentabilidade.

No processo de construção, o grupo é desafiado a conhecer sobre gestão dos resíduos, com visitas a outros ambientes com programas de gestão dos resíduos, subsidiando-se para planejar, executar e monitorar esse processo, com conhecimento. A multiplicação das comunicações tece uma rede social cada vez mais complexa, permitindo o crescimento das comunicações, o desenvolvimento dos indivíduos e a complexidade do social (MORIN, 2005a).

$\mathrm{Na}$ caminhada, O GD aprende que para planejar possíveis ações, mostra-se relevante um diagnóstico prévio da situação dos resíduos, ou seja, conhecer o processo de geração e as condições de seu manejo, da geração, minimização, reaproveitamento, reciclagem, segregação, identificação, acondicionamento, armazenamento, coleta, transporte, tratamento, até o destino final nas diferentes unidades da Instituição (BARROS et al., 2007), proposto através da seguinte estratégia:

[...] Podemos pedir para todos os diretores, através do encaminhamento de um ofício a geração de cada unidade, e como eles fazem hoje com essa geração, assim, teremos esse levantamento... Vamos pedir para o setor de informática colocar no site informações sobre como preencher o solicitado por nós sobre a situação dos resíduos... [...] $\left(\mathrm{GD}_{21}\right)$.

A estratégia de elaboração do diagnóstico proposto não ocorreu, dificuldade que já havia sido prevista por alguns sujeitos. No entanto, foi preciso potencializar essa percepção no grupo e a compreensão de que construir políticas numa perspectiva sistêmica requer a ruptura com a linearidade, ou seja, que um diagnóstico vai além da obtenção de informações possivelmente fragmentadas. Sua realização é uma oportunidade e uma possibilidade de conhecer a realidade local, dificuldades, limites, potencialidades, percepções, conhecimentos e desejos em relação à situação dos resíduos.

Esses limites contribuíram para a evasão de alguns sujeitos do processo educativo construído coletivamente. A persistência necessária na proposta de construção de políticas para a gestão dos resíduos não havia sido suficientemente demonstrada e acatada por todos participantes do grupo, ou seja, alguns aparentemente não se mostravam mais dispostos a construir ações, percepção que foi intensificada pelas dificuldades enfrentadas ao longo do caminho. A aposta na complexidade representa um desafio a quem está disposto a participar da construção do conhecimento de como construir políticas para a gestão dos

Revbea, Rio Grande, 7: 9-15, 2012. 
resíduos mediante um caminho incerto, por isso, não é um pensamento que busca uma resposta aos problemas, às inquietações. É a busca de uma possibilidade de se pensar através da complicação, das incertezas e das contradições (MORIN, 2006).

Avançando na caminhada com o coletivo, a ênfase, predominantemente, era de manifestação e valorização da incerteza nas proposições, num movimento reflexivo sobre o rumo a ser escolhido para a construção de políticas, provocado pelo movimento de desordem: Qual Campus da Instituição apresenta maiores problemas e dificuldades em relação aos resíduos? Por qual tipo de resíduo vamos começar o processo de construção de políticas para a gestão? Que unidade precisa de maior atenção em relação a gestão dos resíduos nesse momento?...(GD22).

O grupo não tinha essas respostas, porém, mediante vivências anteriores de alguns integrantes começaram a emergir estratégias para o encaminhamento da construção de políticas para a gestão dos resíduos:

[...] Pessoal, vamos conhecer a realidade aqui do Campus Capão do Leão, eu sei bem dos problemas daqui de anos, já fui prefeito em uma gestão passada, lembram?... No meu entendimento, é o que tem os maiores problemas em relação aos resíduos [...] $\left(\mathrm{GD}_{2}\right)$.

Essa estratégia fez com que alguns integrantes ampliassem seu conhecimento sobre a realidade do Campus Capão do Leão, sobre os problemas relacionados à disposição final dos resíduos, à identificação de passivo ambiental, desperdícios, manejo inadequado dos resíduos.

Foi demonstrada, no grupo, a preocupação pelos resíduos da área da saúde gerados nas unidades da Instituição, especialmente, pela destinação final adotada, possivelmente causando sérios problemas para o ambiente e a saúde da comunidade, especialmente, àqueles indivíduos pertencentes às unidades geradoras desse tipo de resíduos:

[...] Pelo andamento de nossas reuniões, os resíduos da área da saúde têm prevalecido nossas preocupações, pelas características que predomina esse grupo. Atuamos em unidades que geradoras de resíduo da saúde $[\ldots]\left(\mathrm{GD}_{2}\right)$.

Na medida em que a prática pedagógica resgata a noção sistêmica, o pensamento passa a compreender que a Instituição constitui o todo, e é formada por diversas partes inter-relacionadas; que a construção de políticas na parte interrelaciona-se com o todo e vice e versa.

A necessidade de um maior conhecimento acerca da temática dos resíduos de serviços de saúde, fez com que participantes do grupo realizassem um curso sobre gestão desse tipo de resíduo, para aprender esse saber mais específico, potencializando estratégias à elaboração de políticas para a gestão dos resíduos.

Nesse curso, as vivências socializadas, no coletivo, de aproximação com o conhecimento ocorreram numa dimensão teórica e prática. No aspecto teórico, enfocando conceitos, classificações, cenário da problemática, aspectos éticos, qualidade ambiental; riscos para a saúde e o ambiente; aspectos legais; importância de um plano de gerenciamento dos resíduos; diferentes etapas de um processo de manejo. Já a apreensão do conhecimento na perspectiva prática, foi possibilitada mediante visitas em Instituições de saúde, gerando um diálogo problematizado e crítico com a realidade da Instituição, despertando a curiosidade do coletivo de conhecer uma unidade específica da Instituição: o Hospital Escola. Uma estratégia manifestada por uma integrante foi apresentar ao grupo o programa de gerenciamento dos resíduos de serviços de saúde construído no Hospital Escola: 
"[...] Nós criamos uma comissão lá na minha unidade chamada saúde ambiental, ela é ativa no gerenciamento dos resíduos de serviços de saúde; estamos permanentemente planejando ações para a melhoria de nosso espaço... ...Pessoal, esse é um trabalho que precisa ser contínuo, e acima de tudo educativo [...] $\left(\mathrm{GD}_{4}\right) . "$

Um processo educativo para a construção de políticas para a gestão dos resíduos baseado na incerteza, conduz os sujeitos não somente à dúvida, mas também à estratégia. A incerteza é o fermento do conhecimento, leva a investigar, verificar, comunicar, refletir, inventar (MORIN, 2005a).

\section{CONSIDERAÇÕES FINAIS}

Para construir políticas para a gestão dos resíduos na perspectiva da educação ambiental, mostrou-se fundamental potencializar modelos de aprendizagem que provocassem a desacomodação permanente, instigando a reforma do pensamento dos sujeitos, valorizando a importância de sua participação, encaminhando para uma percepção multidimensional da realidade. Esse reconhecimento teve implicações, também, na compreensão a respeito da dinâmica da realidade e da vida, implicações na maneira de ser, de fazer, de viver/conviver coletivamente, propiciando um novo reposicionamento diante do mundo e da vida.

\section{REFERÊNCIAS}

BARROS, R. M.; et al. Diagnóstico da gestão dos resíduos no campus universitário da UNIFEI/MG. In: CONGRESSO BRASILEIRO DE ENGENHARIASANITÁRIAEAMBIENTAL, 24, 2007. Belo Horizonte. Anais... Belo Horizonte, 2007. 1 CD-ROM.

CORRÊA, L. B. Construção de políticas para a gestão dos resíduos em uma instituição de ensino superior na pers pectiva da educação ambiental. 2009. 286 f. Tese (Doutorado em Educação Ambiental) - Universidade Federal do Rio Grande, Rio Grande (RS), 2009.

DE CONTO, S. M.; SILVA, E. J. A.; PESSIN, N. Solid waste management practice as subsidize for the environmental management system at higher education institutions: case study at the university of Caxias do Sul. In: SIMPOSIO INTERNAZIONALE DI INGENERIA SANITARIAAMBIENTALE, 2004. Taormina. Proceedings... Taormina: ANDIS, 2004. 1 CDROM.

DIAS, G. F. Educação ambiental: princípios e práticas. 7. ed. São Paulo: Gaia, 2001. $551 \mathrm{p}$.

. Educação e gestão ambiental. São Paulo: Gaia, 2006. 118p.

JACOBI, P. R. Educar para a sustentabilidade: complexidade, reflexividade, desafios. Educação e Pesquisa, v. 31, n. 2, p. 233-250, maio/ago., 2005.

MORAES, R. Uma tempestade de luz: a compreensão possibilitada pela análise textual discursiva. Ciência \& Educação, v. 9, n. 2, p. 191-211, 2003.

MORIN. O método 3: o conhecimento do conhecimento. Porto Alegre: Sulina, 2005a. $286 \mathrm{p}$.

O método 6: ética. Porto Alegre: Sulina, 2005b. 222p.

. Introdução ao pensamento complexo. Porto Alegre: Sulina, 2006. 120p. 
CIURANA, E. R.; MOTA, R. D. Educar na era planetária: o pensamento complexo como método de aprendizagem no erro e na incerteza humana. São Paulo: Cortez, 2003. $111 \mathrm{p}$.

QUINTAS, J. S.; GOMES, P. M.; UEMA, E. E. Pensando e praticando a educação no processo de gestão ambiental: uma concepção pedagógica e metodológica para a prática da educação ambiental no licenciamento. Brasília: IBAMA, 2005.

THIOLLENT, M. Metodologia da pesquisa-ação. 16 ed. São Paulo: Cortez, 2008. 132p. 